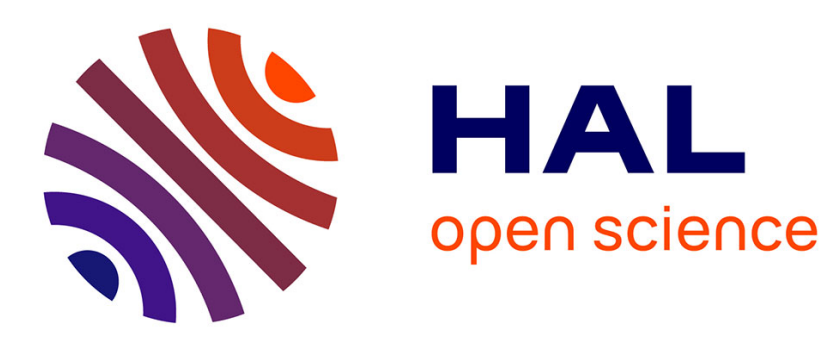

\title{
Simulation of Road Surface Roughness Based on the Piecewise Fractal Function
}

\author{
Zhixiong Lu, Lanying Zhao, Xiaoqin Li, Jun Yuan
}

\section{To cite this version:}

Zhixiong Lu, Lanying Zhao, Xiaoqin Li, Jun Yuan. Simulation of Road Surface Roughness Based on the Piecewise Fractal Function. 4th Conference on Computer and Computing Technologies in Agriculture (CCTA), Oct 2010, Nanchang, China. pp.294-305, 10.1007/978-3-642-18369-0_34 . hal01564854

\section{HAL Id: hal-01564854 \\ https://hal.inria.fr/hal-01564854}

Submitted on 19 Jul 2017

HAL is a multi-disciplinary open access archive for the deposit and dissemination of scientific research documents, whether they are published or not. The documents may come from teaching and research institutions in France or abroad, or from public or private research centers.
L'archive ouverte pluridisciplinaire HAL, est destinée au dépôt et à la diffusion de documents scientifiques de niveau recherche, publiés ou non, émanant des établissements d'enseignement et de recherche français ou étrangers, des laboratoires publics ou privés. 


\title{
Simulation of Road Surface Roughness Based on the Piecewise Fractal Function
}

\author{
Zhixiong $\mathrm{Lu}^{1}$, Lanying $\mathrm{Zhao}^{2}$, Xiaoqin $\mathrm{Li}^{1}$, Jun Yuan ${ }^{1}$ \\ ( ${ }^{1}$ Engineering Collage, Nanjing Agricultural University. Nanjing, 210031, Nanjing, Chinese; \\ ${ }^{2}$ Institute of mechanical engineering, Dalian Ocean University, 116023,Dalian,Chinese) \\ \{ Zhixiong Lu, Lanying Zhao, Xiaoqin Li, Jun Yuan, luzx@njau.edu.cn \}
}

\begin{abstract}
The fractal interpolation theory was used to the research of road surface roughness, and its simulating method was proposed through the piecewise fractal model. The fractal interpolation model was established for the actual road surface roughness, its influential factors and their laws of influencing were also analyzed, and then the model accuracy was confirmed by the fractal parameters and the traditional parameters. The research indicated that the simulated parameters of road surface in different resolutions and the traditional parameters can keep better consistency with the actual road surface. The contraction factor and the road surface sampling resolution are main influential factors of the model. The contraction factor's selection decided directly the fractal dimension of the simulated road, and the simulated precision reduced with the decrease of sample resolution at the same time. In fact, this method is able to obtain a better simulation results, as long as the sample resolution was restrained on the scaleless range of road surface roughness.
\end{abstract}

Keywords: Road surface roughness; Piecewise Fractal model; Contraction factor; Scaleless range

\section{Introduction}

Road surface longitudinal elevation change is a non-stationary random process with multi-scale features. Therefore road roughness resolution, measured by instrument with a certain resolution, reflects the roughness in the limited level rather than completely and really, which manifests scale correlation of road roughness. Obviously, statistical characterization parameters have certain deviation, when the data was collected by road roughness testers with different resolution, and this deviation will affect the evaluation on road surface roughness and the accuracy of analysis on vehicle vibration response. But all these questions will be solved, if the limited test data can be used to simulate a more precise road vertical elevation.

At present, roughness testers collect longitudinal elevation data of road surface along the vehicles travel direction, which is in certain sampling interval. So the resolution cannot be high enough to meet the require mends achieve of line survey, and the smooth curves, fitted by data tested directly or obtained by the traditional interpolation methods (the Lagrange interpolation, transect interpolation and so on), 
have good fitting effect, but can not characterize self-similar surface roughness characteristics and fine structure. In this paper, fractal interpolating method was introduced to simulate road roughness with fractal characteristics, and simulative results were verified and analyzed in detail.

\section{Paper Preparation}

\section{1 self-affine fractal interpolation}

The basis of fractal interpolation theory is iteration function systems (IFS).IFS consists of a set of limited contraction mapping family on a complete metric space $(W i: X \rightarrow X, i=1,2, \ldots, \mathrm{N}$ ) and the corresponding contraction factor $S i \in[0,1]$. Denoted $\{X ; W i, i=1,2, \ldots, \mathrm{N}\}$, the contraction factor $S=\max \{S i, i=1$, $2, \ldots, \mathrm{N}\}$.

Fractal interpolation actually constructed a iteration function system, the attractor is the graphical of interpolation function. To the two-dimensional discrete $\operatorname{data}\left\{\left(\mathrm{x}_{\mathrm{i}}, \mathrm{y}_{\mathrm{i}}\right), \mathrm{i}=0,1, \ldots, \mathrm{N}\right\} \in \mathrm{R}^{2}$, fractal interpolation function is a continuous function $\mathrm{f}(\mathrm{x}), \mathrm{x} \in\left[\mathrm{x}_{0}, \mathrm{x}_{\mathrm{n}}\right] \rightarrow \mathrm{R}$, which interpolated to $\left\{\left(\mathrm{x}_{\mathrm{i}}, \mathrm{y}_{\mathrm{i}}\right), \mathrm{i}=0,1, \ldots, \mathrm{N}\right\}$, and for each $\mathrm{i}$, function $\mathrm{f}(\mathrm{x})$ meet with $\mathrm{f}\left(\mathrm{x}_{\mathrm{i}}\right)=\mathrm{y}_{\mathrm{i}}$, the graph is attractors of a doublecurved iterated function system .

By constructed a iterated function system $\left\{\mathrm{R}^{2}: W i, i=1,2, \ldots, \mathrm{N}\right\}$ which attractor is equal to the graph of interpolation $\mathrm{f}(\mathrm{x})$, its constructed method as follows: suppose each of iterated function systems $W i$ is the affine transformation $W i$ : $\mathrm{R}^{2} \rightarrow \mathrm{R}^{2}$, then

$$
W_{i}\left(\begin{array}{l}
x \\
y
\end{array}\right)=\left(\begin{array}{l}
a_{i} 0 \\
c_{i} d_{i}
\end{array}\right)\left(\begin{array}{l}
x \\
y
\end{array}\right)+\left(\begin{array}{l}
e_{i} \\
f_{i}
\end{array}\right)_{, i=1,2, \ldots, \mathrm{N}}
$$

And satisfy the conditions:

$$
W_{i}\left(\begin{array}{l}
x_{0} \\
y_{0}
\end{array}\right)=\left(\begin{array}{l}
x_{i-1} \\
y_{i-1}
\end{array}\right), W_{i}\left(\begin{array}{l}
x_{n} \\
y_{n}
\end{array}\right)=\left(\begin{array}{l}
x_{i} \\
y_{i}
\end{array}\right), i=1,2, \ldots, \mathrm{N}
$$

$a_{i}, c_{i}, d_{i}, e_{i}, f_{i}$ is the real coefficients to determine an affine transformation, by (2) formula to determine the following four linear equations. 


$$
\left\{\begin{array}{l}
a_{i} x_{0}+e_{i}=x_{i-1} \\
a_{i} x_{n}+e_{i}=x_{i} \\
c_{i} x_{0}+d_{i} y_{0}+f_{i}=y_{i-1} \\
c_{i} x_{n}+d_{i} y_{n}+f_{i}=y_{i}
\end{array}\right.
$$

Under the constrained condition, here given only four equations, however ,five variables, so there is a free variable, generally take $\mathrm{d}_{\mathrm{i}}$ as the free variable and call it the vertical scale factor , limited $0 \leq \leq d_{i \mid \leq 1}(\mathrm{i}=0,1, \ldots, \mathrm{N})$, which is determined by the property of the affine transform. Here let $\mathrm{d}_{\mathrm{i}}=0$, make the iteration function system $W i$ always map the line which parallel to the y-axis still to the line which parallel to the y-axis. Then $\mathrm{d}_{\mathrm{i}}$ express the transform proportion. If $d_{i}=0$, gain the piecewise linear interpolation function. When $d_{i}$ is arbitrary, combination of the above formula, the other factors of the iterated function system can be derived, as follows:

$$
\begin{gathered}
a_{i}=\left(x_{i}-x_{i-1}\right) /\left(x_{n}-x_{0}\right) \\
c_{i}=\left(y_{i}-y_{i-1}\right) /\left(x_{n}-x_{0}\right)-d_{i}\left(y_{n}-y_{0}\right) /\left(x_{n}-x_{0}\right) \\
e_{i}=\left(x_{n} x_{i-1}-x_{0} x_{i}\right) /\left(x_{n}-x_{0}\right) \\
f_{i}=\left(x_{n} y_{i-1}-x_{0} y_{i}\right) /\left(x_{n}-x_{0}\right)-d_{i}\left(x_{n} y_{0}-x_{0} y_{n}\right) /\left(x_{n}-x_{0}\right)
\end{gathered}
$$

Barnsley ${ }^{[1]}$, etc ,who have prove ,IFS which constructed by this method always have displacement attractor, which must be a continuous function graphics, and also through the interpolation points, this continuous function is the fractal interpolation functions.

\subsection{Recurrent fractal interpolation}

Piecewise linear fractal interpolation method is the promotion of linear fractal interpolation. The basic idea is :put the original curve into several paragraphs, which equal or sub-ranges coordinates along the $\mathrm{x}$-axis direction, or several consecutive interpolation points can be put in a group, then each piece of curve according to above fractal interpolation methods get fitting curve ${ }^{[2]}$. Piecewise fractal interpolation is a new fitting method of data, change its parameters, can get the interpolation curve with different dimensions, generated curve in each segment have an independent selfsimilarity and fractal dimension. 


\section{The algorithm of vertical scale factor}

Based on constructed curve on fractal interpolation function, fractal dimension D is determined by vertical scale factor ${ }^{d}$. If known data $\{(\mathrm{xi}, \mathrm{yi}) ; \mathrm{i}=0,1, \ldots, \mathrm{n}\}$ isn't collinear, then, D's value is the only solution of equation $\sum_{i=1}^{n}\left|d_{i}\right| a_{i}^{D-1}=1$;if interpolation points collinear, $\mathrm{D}=1$; under the conditions of $0 \leqslant \mid d_{i} \leqslant 1$, appropriately select ${ }_{i}$,make D arbitrarily close to 2 . however ,in the linear fractal interpolation computations, given random the vertical scale factor $d_{i}$, values, it is difficult to make the fractal curve by interpolation more accurately approximate to the real curve or discrete points.

According to the principles of above fractal interpolation, be given a set of $d_{i}$, values for each, an iterated function system can be completely determined, this iterated function system attractor image is the interpolation image through the known points, how to choose the optimal vertical scale factor to control the shape of the fractal curve, make it possible approximate to the original objective function or discrete data points, which is called the inverse problem of fractal interpolation ${ }^{[3]}$. Determination of the optimal $d_{i}$ value is difficult, Mazel and Hayes ${ }^{[4]}$ give the geometric and analytical methods by using fractal interpolation function to fit discrete data, The geometric and analytical methods is the classic algorithm to calculate vertical scale factor. This paper use particle swarm optimal algorithm to achieve the optimization of vertical scale factor.

\section{Fractal interpolation of road surface roughness}

As to collected original elevation data of road roughness in the form of discrete points, usually record data by directly connect or traditional interpolation method, then calculate the corresponding deviation index, such as the root mean square, power spectral density and the international roughness index, because of road testing instrument resolution, the evaluation results only reflect the roughness of some cases. In the case of retention measured discrete data, it is the purpose of fractal interpolation of the road roughness original elevation discrete data which obtain the road roughness information between the sampling points, make the fractal interpolation curve far more approximation to the actual road longitudinal curve.

According to preliminary studies, we know that road roughness has obvious fractal characteristics. Compared to several thousand kilometers road surface, the longitudinal elevation data to evaluate the road roughness be collected by surface detection equipment, which be limited by hard disk storage space of the detection equipment, usually only a small part of road data, therefore, on the research that the 
road roughness data may not have the self-affine properties or similar self-affine properties, fractal interpolation on the data may produce large errors ${ }^{[5,6]}$. Therefore, we simulated the road roughness elevation data adapting piecewise fractal interpolation method.

\subsection{Fractal Interpolation Simulation of Road Roughness}

In this paper, vertical elevation data of asphalt concrete road surface roughness is the object of the study, experimental data collected is the sequence of discrete points: $\{(\mathrm{n}, \mathrm{yn}), \mathrm{n}=0,1, \ldots, \mathrm{N}\}, \mathrm{N}$ is the sampling points, sampling interval of instrument is $0.16 \mathrm{~m}$ (resolution), 1024 data points is obtained, then the sample length is $1024 *$ $0.16=163.84 \mathrm{~m}$. The measured data were displayed in figure 1 . In this sample paragraph, points were taken in every 20 point and interval $3.2 \mathrm{~m}$, where lowresolution data ( figure 2) is obtained.

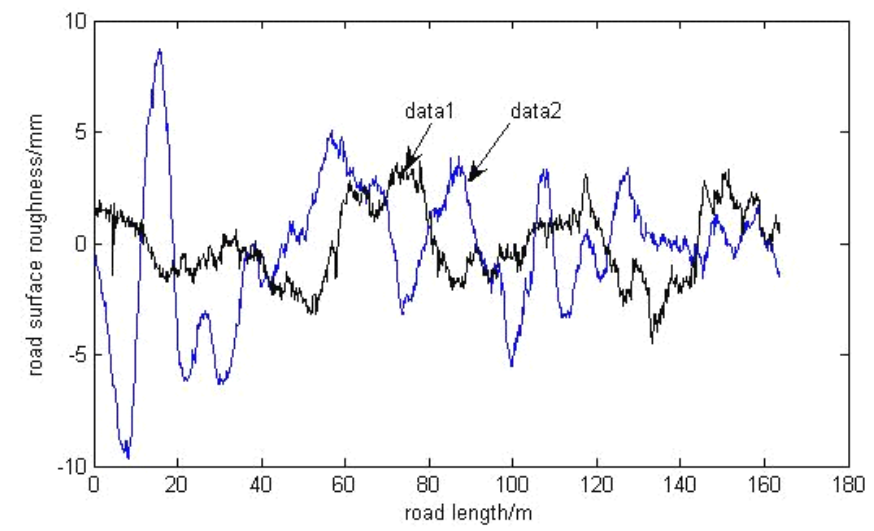

Fig. 1. The measured road surface with high resolution

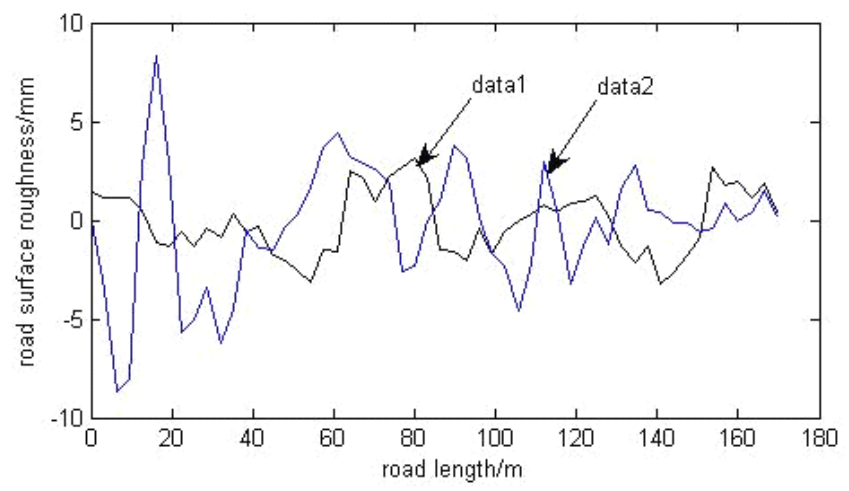

Fig. 2. The measured road surface with low resolution 
According to the fractal interpolation theory and the vertical scale factor values estimated by analytic method, the result that high-resolution and low resolution data in Figure 2 is sub-fractal interpolated is shown in Figure 3, the algorithm procedure is implemented by MATLAB7.0.

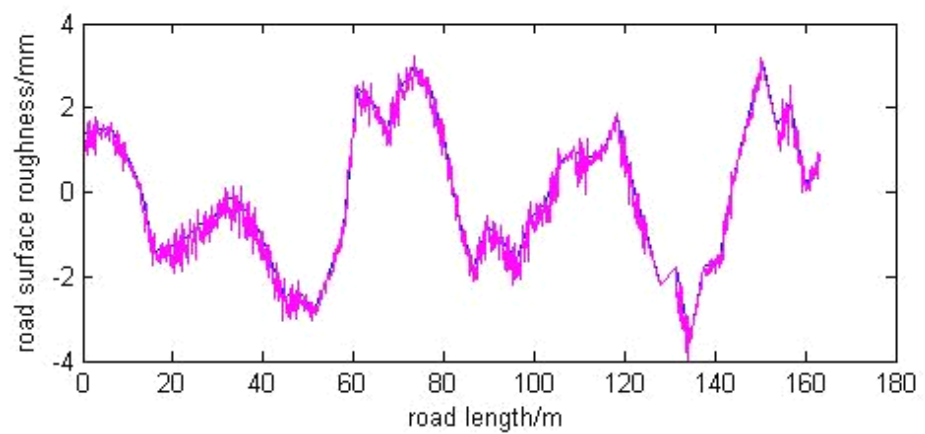

a)

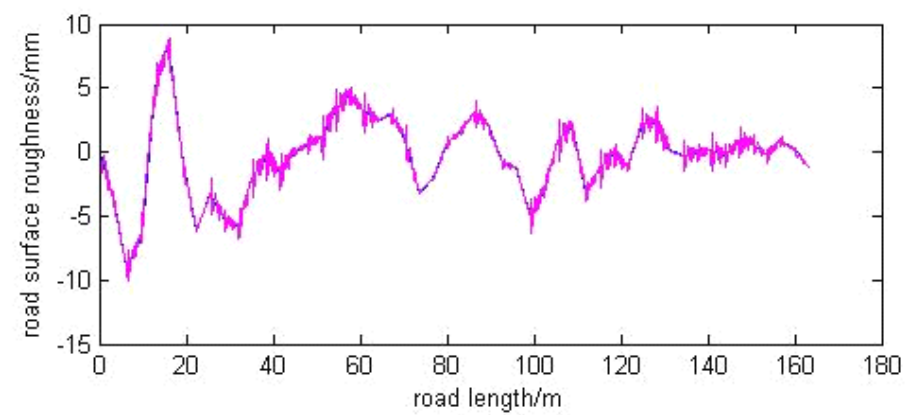

b)

Fig. 3. The fractal interpolation road surfaces: a) Interpolation of low-resolution data1, b) Interpolation of low-resolution data2.

\subsection{Validity of Fractal Interpolation}

In order to test the validity of the results of fractal interpolation, the curves of the characterization of road roughness parameters(root mean square deviation of profile, fractal dimension,) showed in Figure 1 and Figure 3 is calculated and the values is shown in Table 1, in which D is calculated by root mean square method. Fractal characteristics of curve of the original data and simulation of fractal interpolation were compared in Figure 4. 
Table 1. The values of several characterizing parameters of measured and interpolated road surface

\begin{tabular}{lcccc}
\hline \multirow{2}{*}{ parameter } & data1 & \multicolumn{3}{c}{ data2 } \\
\cline { 2 - 5 } & $\begin{array}{c}\text { Measured } \\
\text { road surface }\end{array}$ & $\begin{array}{c}\text { Simulated } \\
\text { road surface }\end{array}$ & $\begin{array}{c}\text { Measured } \\
\text { road surface }\end{array}$ & $\begin{array}{c}\text { Simulated } \\
\text { road surface }\end{array}$ \\
\hline $\mathrm{R}_{\mathrm{q}}$ & 1.6606 & 1.6048 & 3.1184 & 3.0877 \\
$\mathrm{D}$ & 1.6298 & 1.6174 & 1.4732 & 1.4726 \\
\hline
\end{tabular}
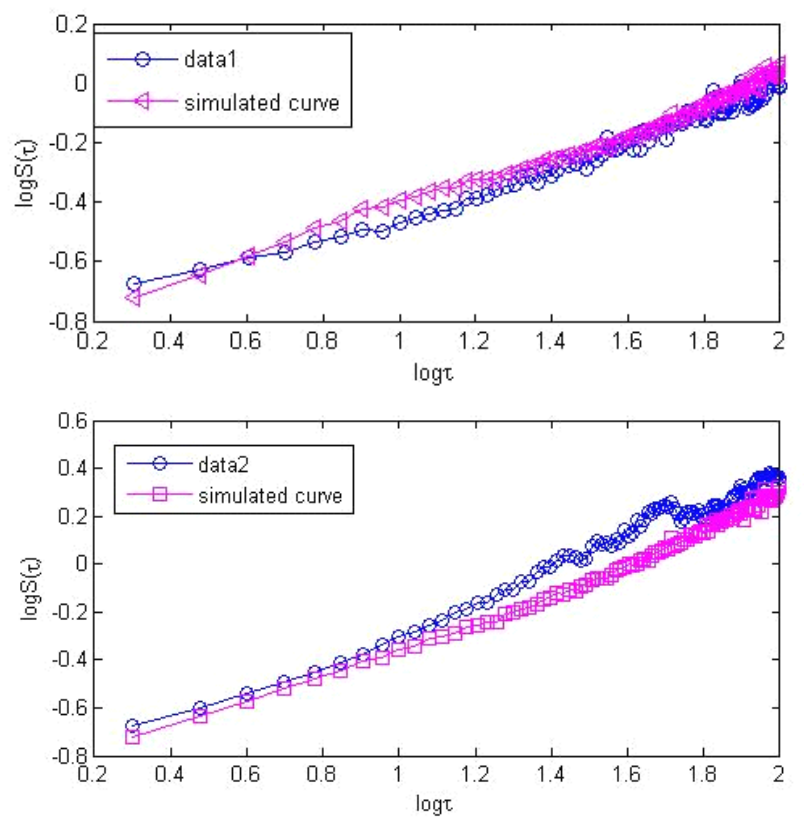

Fig. 4. Comparison of primal data and interpolation curve

Comparison with fractal characteristics of curve of the original data and simulation of fractal interpolation in Figure 4 can be found: (1) scaling law relation between simulation curve obtained in the first interpolation interval scale in Figure 4 a) with the original data has crossover phenomenon. It is known by calculating the number of dimensions : the fractal dimensions are still relatively close, both of which fractal error is $0.76 \%$; Scaling law relation between simulation curve obtained in the first interpolation interval scale in Figure $4 \mathrm{~b}$ ) with the original data is very close. This could explain that the simulation of surface roughness by the fractal interpolation function can be appreciatively simulated with the original data, self-similarity.

(2) Time-domain characterization (root mean square deviation of profile parameters) of fractal interpolation curve and the measured road roughness curve are very close, indicating the two have good statistical self-similarity.

The above two points can determine that fractal characteristics simulation of road roughness by the fractal interpolation function is feasible.

Compared with the measured surface and the interpolated surface graphics in 
Figure 1 and Figure 3 and the corresponding characterization parameters in table 1, it can be seen that representation parameter values of the measured fractal interpolation curve and surface roughness data graph is very close, both of which are very good Statistical self-similarity, indicating that sub-fractal interpolation function with the road roughness of fractal characteristics is a sound result.

\subsection{The accuracy factor analyses affecting the fractal interpolation of road surface roughness}

Vertical scale factor is the key factor to control the accuracy of the fractal interpolation, which has been described in principle. At the same time, changes in the number of iterations, the size of the sampling point interval (resolution) and sampling length also affect the fractal interpolation results.

\subsubsection{Vertical scale factor}

According to the requirements principles of fractal interpolation on the vertical scale factor, different ${ }^{d_{i}}$ values is selected in the condition: $0 \leq\left|d_{i}\right| \leq 1$, In order to analyze directly the vertical scale factor on the effect of the interpolation, this method of direct value is used, which are taken $d_{i}=0.05, d_{i} \in(0,0.2), d_{i}=0.2$, $d_{i} \in(0,1)$ the measured data (Figure 1 data1) Figure 20 points sampling interval) are fractal interpolated, interpolation process selects the same number of iterations (interpolation results Figure omitted). Root mean square method is used to analyze fractal characteristics of the interpolated curve of shown in Figure 5, the characterization parameter of fractal interpolation curve calculation is shown in table 2 below.

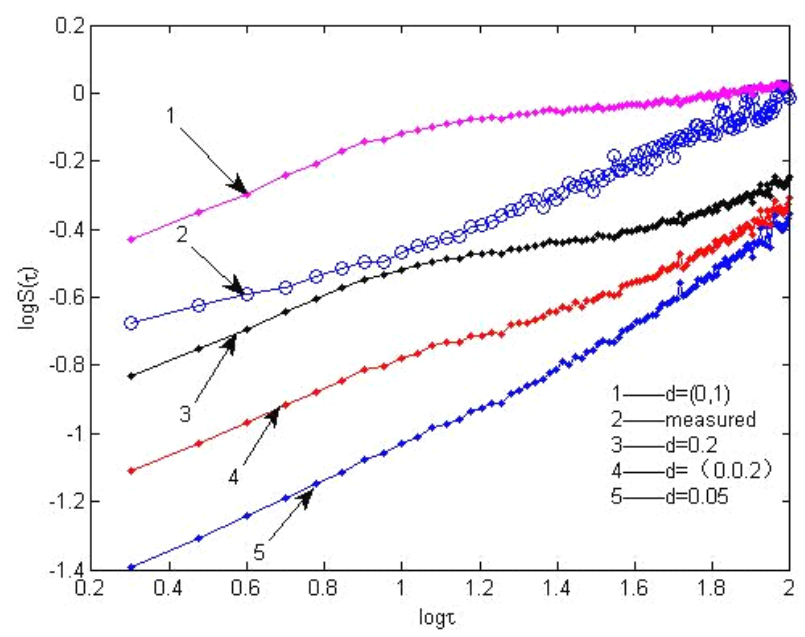

Fig. 5. Fractal plots of interpolation curves with different vertical scale factor 
Table 2. Comparison of parameters with different $\mathrm{d}_{\mathrm{i}}$

\begin{tabular}{llllll}
\hline parameters & $\begin{array}{l}\text { Measured } \\
\text { data }\end{array}$ & $\begin{array}{c}d_{i} \\
=0.05\end{array}$ & $d_{i \in(0,0.2)}$ & $\begin{array}{c}d_{i} \\
=0.2\end{array}$ & $d_{i \in(0,1)}$ \\
\hline $\begin{array}{l}\text { root mean } \\
\text { square }\end{array}$ & 1.6606 & 1.5931 & 1.6299 & 1.6453 & 1.8443 \\
$\begin{array}{l}\text { fractal } \\
\text { dimension }\end{array}$ & 1.6298 & 1.4081 & 1.6113 & 1.6093 & 1.5698 \\
\hline
\end{tabular}

From Figure 5, it can be found that the simulated curves obtained by interpolation in the first scale have good fractal characteristics, and the slopes are close, but with the selected vertical scale factor increasing, the scaling law curve in the double logarithmic coordinates of the intercept increases, therefore, the physical meaning of the root mean square method calculating fractal dimension can explain the vertical scale factor is directly related to the absolute measure of the value of road roughness size, which is the important factor to effect fractal interpolation. In the measured surface roughness simulation of fractal interpolation, it is needed to consider the vertical scale factor.

From comparison characterization parameters between measured data and simulated curves under different vertical scale factor in Table 2, it shows that when $d_{i=0.2 、} d_{i} \in(0,0.2)$, root mean square deviation by the interpolation curve is close to that by the measured curve, which is consistent with the Analysis of Fractal Interpolation scaling law curve in double logarithmic coordinates $\log S(\tau)$ and $\log \tau$ in the size of the intercept in Figure 5 and the vertical scale factor values greater, RMS deviation of surface roughness greater and the road more rough. the fractal dimension of the first scale range calculated by RMS is also small difference with the measured data, therefore, when the measured surface roughness is simulated by the fractal interpolation, the vertical scale factor in its allowable range can be neither too large nor too small.

\subsubsection{Sampling interval}

To be Convenient for the comprehensive analysis, measured road roughness in Figure $1 \mathrm{a}$ ) is still as the interpolation object. Sampled low-resolution data on road roughness are based on interval 10, 20, 50 points, Resolution $\mathrm{R}$ were: $\mathrm{R}=1.6 \mathrm{~m}, \mathrm{R}=$ $3.2 \mathrm{~m}, \mathrm{R}=8 \mathrm{~m}$, then take the respective analytical method to calculate the value of the vertical scale factor and simulate discrete data points based on these low-resolution data by fractal interpolation, the result of fractal interpolation simulation of the road roughness is shown in Figure 6. Fractal plots of interpolation curves with different $\mathrm{R}$ were shown in figure 7. 


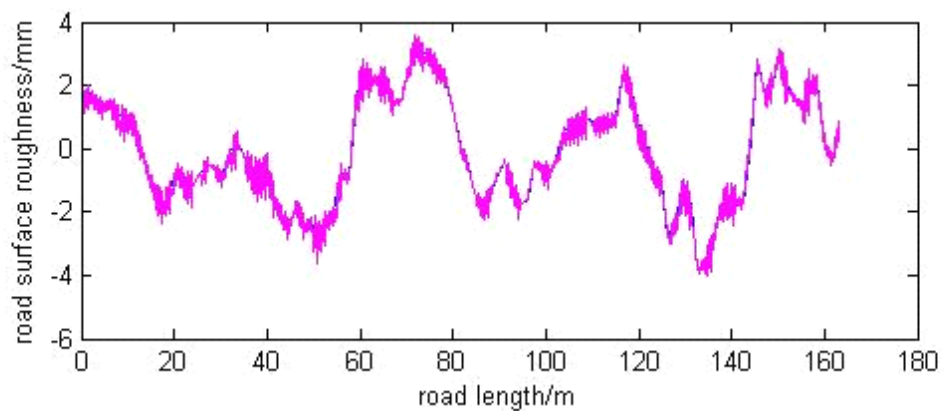

a)

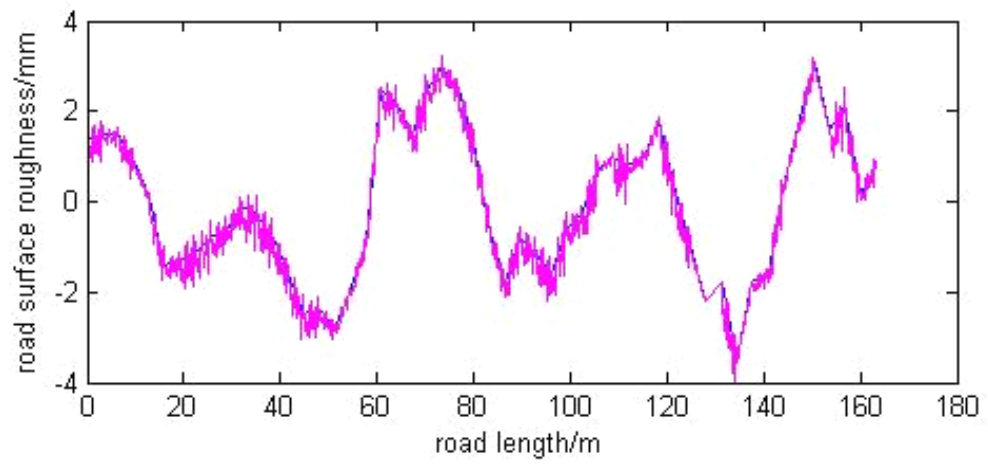

b)

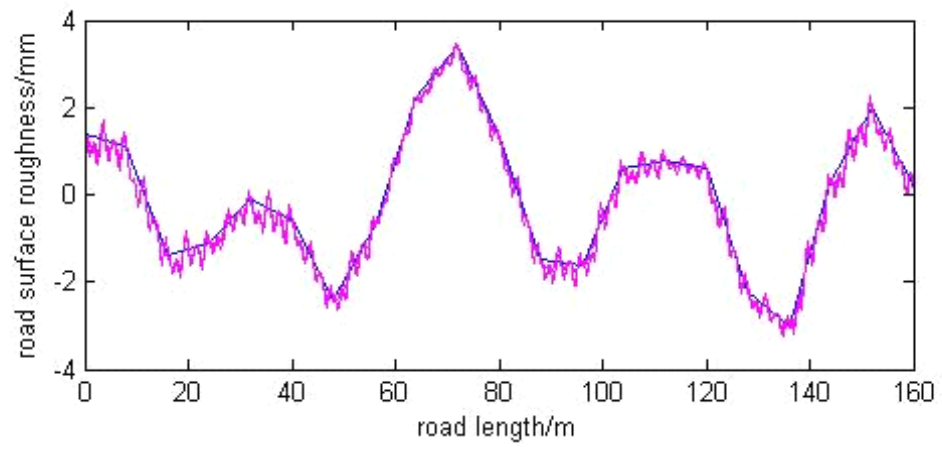

c)

Fig. 6. Effects of resolution of original data to interpolation result. a) $R=1.6 \mathrm{~m}$; b) $R=3.2 \mathrm{~m}$; c) $R=8 \mathrm{~m}$ 


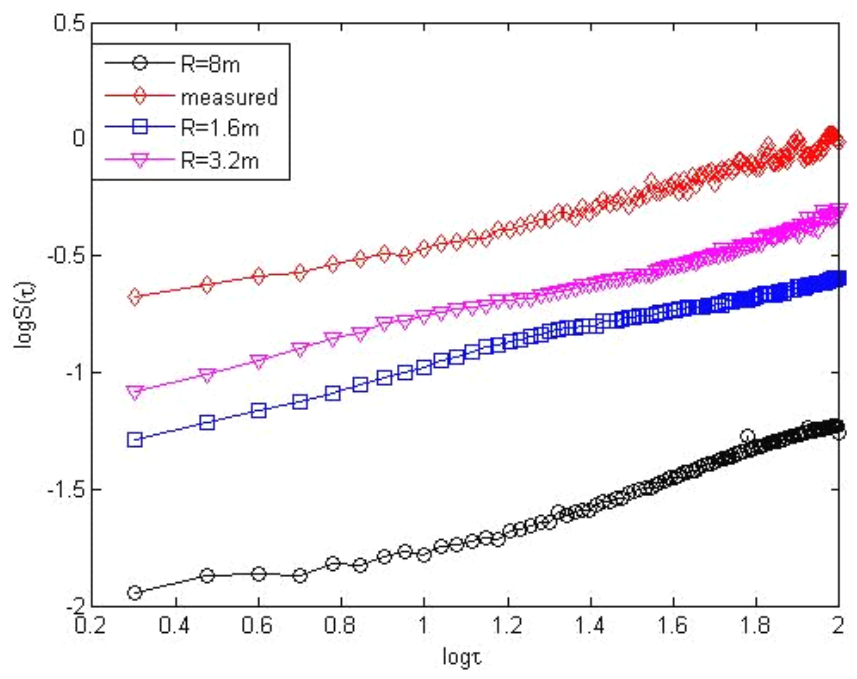

Fig. 7. Fractal plots of interpolation curves with different $R$

From the figure 7 , we can see that the scaling law of the fractal interpolation curve is greater with the high-resolution than with the low-resolution. The meticulous structures of the simulated curve will reduction, when the resolution is too low according the figure 6 .

Theoretically the sampling interval is smaller is better. In fact, the sampling interval obtains is too small, does not have affects too greatly to increases the scaleless range and the computation fractal dimension value, because of the gathering system's precision or the resolution limit. According to the fractal interpolation theory, so long as the sampling interval in the non-scale sector, the good simulation effect can be obtained with the reasonable influence parameter. But because errors of measure, the vertical scale factor counting and the limit of times of the iterative, the sampling interval is smaller than the one third of scaleless range.

\subsubsection{The Sampling length and number of iterations}

Because the curve interpolated in too long curve segment does not have the same as fractal law of the actual road roughness, when the sample length of the original data curve is too large, piecewise interpolation should be needed, each should be less than the length of the largest scale of a fractal rough surface characteristic.

According to the definition of fractal interpolation, with the number of iterations increasing, the number of interpolation points is increased to $M$ times every time ( $\mathrm{M}$ is the number of interpolation points) which is based on the rules of affine transformation. With the increase of the number of iterations, when the interpolation points are enough, the graph of the attractors of iterated function systems is established by the intensive interpolation points. Therefore, when the selected couples of original data interpolation points are less, which the sampling interval is large, it should increase the number of the iteration to generate more points so as to make a 
interpolation graph structure more precise. However, when the number of iterations increases, the computation also increases and the run time is too long. So when the calculation process, the sample length, sampling interval and vertical scale factor are confirmed, to adjust appropriately the number of iterations to get good simulation results is to avoid difficulties in the cost of computation taken place by the infinite number of iterations.

\section{Conclusion}

Fractal interpolation, which is based on affine transformation iteration function system, can fully reflect the self-similarity structure 。Based on vertical elevation discrete data of road surface roughness, continuous simulation curves are produced through fractal interpolation simulation, and these curves not only retain the information of original discrete points but also characterized more detailed information on road roughness between the discrete points, so instrumentations' resolution of road roughness could been reduced.

From the preparation of characterizations of fractal interpolation curve and the measured road roughness curve, we can see that the application of piecewise fractal function was feasible in self-similarity simulation of the measured road surface with low resolution.

The accuracy of the road fractal interpolation is mainly decided by the computation of the vertical scale factor. At the same time, the iterative number of times and the primary data sampling interval (i.e. resolution) also affects the effect of road fractal interpolation.

\section{Reference}

1. Barnsley M.: Fractals everywere. New York: Academic Press, 207-233(1988)

2. STAMPS A E.: Fractals, skylines, nature and beauty. Landscape and Urban Planning, 60,163184(2002)

3. Sha Zhen.: A class of functional equation and fractal interpolation function, Appl.Math. JCU, 14B, 90-98(1999)

4. David S. Mazel. Monson H Hayes.: Using iterated function systems to model discrete sequences.IEEE Transactions on signal processing. 40(7), 1724-1734(1992)

5. Xie H, Sun H, Ju Y, Feng Z.: Study on Generation of Rock Fracture Surface by Using Fractal Interpolation. Int J of Solids \& Structures, 38,5765-5787 (2001)

6. Ruan Huojun, Sha Zhen, Su Weiyi.: Parameter identification problem of the fractal interpolation functions. Numerical MathmaticsA. Journal of Chinese Universities, 12(2), 205-213(2003) 